\title{
Optimally Sparse Image Representation by the Easy Path Wavelet Transform
}

\author{
Gerlind Plonka ${ }^{1}$, Stefanie Tenorth ${ }^{1}$ and Armin Iske ${ }^{2}$ \\ ${ }^{1}$ Institute for Numerical and Applied Mathematics, \\ University of Göttingen, Lotzestrße 16-18, 37083 Göttingen, Germany \\ plonka@math. uni-goettingen.de, s.tenorth@math.uni-goettingen.de \\ 2 Department of Mathematics, University of Hamburg, 20146 Hamburg, Germany \\ iske@math. uni-hamburg.de
}

\begin{abstract}
The Easy Path Wavelet Transform (EPWT) [20] has recently been proposed by one of the authors as a tool for sparse representations of bivariate functions from discrete data, in particular from image data. The EPWT is a locally adaptive wavelet transform. It works along pathways through the array of function values and it exploits the local correlations of the given data in a simple appropriate manner. In this paper, we show that the EPWT leads, for a suitable choice of the pathways, to optimal $N$-term approximations for piecewise Hölder continuous functions with singularities along curves.
\end{abstract}

Key words. sparse data representation, wavelet transform along pathways, image data compression, adaptive wavelet bases, $N$-term approximation

AMS Subject classifications. 41A25, 42C40, 68U10, 94A08

\section{Introduction}

During the last few years, there has been an increasing interest in efficient representations of large high-dimensional data, especially for signals. In the one-dimensional case, wavelets are particularly efficient to represent piecewise smooth signals with point singularities. In higher dimensions, however, tensor product wavelet bases are no longer optimal for the representation of piecewise smooth functions with discontinuities along curves.

Just very recently, more sophisticated methods were developed to design approximation schemes for efficient representations of two-dimensional data, in particular for images, where correlations along curves are essentially taken into account to capture the geometry of the given data. Curvelets $[2,3]$, shearlets $[12,13]$ and directionlets [26] are examples for non-adaptive highly redundant function frames with strong anisotropic directional selectivity.

For piecewise Hölder continuous functions of second order with discontinuities along $C^{2}$-curves, Candès and Donoho [2] proved that a best approximation $f_{N}$ to a given function 
$f$ with $N$ curvelets satisfies the asymptotic bound

$$
\left\|f-f_{N}\right\|^{2} \leq C N^{-2}\left(\log _{2} N\right)^{3},
$$

whereas a (tensor product) wavelet expansion leads to asymptotically only $\mathcal{O}\left(N^{-1}\right)$ [18]. Up to the $\left(\log _{2} N\right)^{3}$ factor, this curvelet approximation result is asymptotically optimal (see [9], Section 7.4). A similar estimate has been achieved by Guo and Labate [12] for shearlet frames. These results, however, are not adaptive with respect to the assumed regularity of the target function, and so they cannot be applied to images of less regularity, i.e., images which are not at least piecewise $C^{2}$ with discontinuities along $C^{2}$-curves.

In such relevant cases, one should rather adapt the approximation scheme to the image geometry, instead of fixing a basis or a frame beforehand to approximate $f$. During the last few years, several different approaches were developed for doing so $[1,7,8,10,11,14,15$, $17,19,20,21,23,24,25]$. In [17], for instance, bandelet orthogonal bases and frames are introduced to adapt to the geometric regularity of the image. Due to their construction, the utilized bandelets are anisotropic wavelets that are warped along a geometrical flow to generate orthonormal bases in different bands. LePennec and Mallat [17] showed that their bandelet dictionary yields asymptotically optimal $N$-term approximations, even in more general image models, where the edges may also be blurred.

Further examples for geometry-based image representations are the nonlinear edgeadapted (EA) multiscale decompositions in $[1,14]$ (and references therein), being based on ENO reconstructions. We remark that the resulting ENO-EA schemes lead to an optimal $N$-term approximation, yielding $\left\|f-f_{N}\right\|^{2} \leq C N^{-2}$ for piecewise $C^{2}$-functions with discontinuities along $C^{2}$-curves. Moreover, unlike previous non-adaptive schemes, the ENO-EA multiresolution techniques provide optimal approximation results also for $B V$ spaces and $L^{p}$ spaces, see [1]. For piecewise Hölder continuous functions of order $\alpha<1$ with discontinuities along curves, also an adaptive wavelet approximation with isotropic wavelets can lead to optimal $N$-term approximation, see [9] and [16].

In many relevant applications to image denoising and image regularization, the space $B V$ containing all functions of bounded variation plays an important role. The space $B V$ seems to be well-adapted to model natural images, since it also allows sharp edges, unlike the bivariate Besov space $B_{1}^{1}\left(L_{1}\right)$, see [4]. However, in case of $B V$ spaces, there is no simple data representation, e.g. in terms of wavelet coefficients. For useful relations between Haar decompositions on dyadic rings and the space BV we refer to [5].

In this paper, we prove optimal $N$-term approximation for piecewise Hölder continuous functions with singularities along curves using the locally adaptive Easy Path Wavelet Transform (EPWT) which has recently been explored in our previous paper [20]. The EPWT applies a one-dimensional wavelet transform along suitable pathways of data vectors, where local correlations of the given data are essentially exploited. As supported by our numerical experiments in [20], the EPWT leads to an efficient compression method for two-dimensional digital data, especially for image data. In this paper, we focus on the approximation properties of the EPWT. We show that the application of the EPWT leads to the optimal $N$-term approximation of the form

$$
\left\|f-f_{N}\right\|_{2}^{2} \leq C N^{-\alpha}
$$

for piecewise Hölder continuous functions of order $\alpha$ (with $0<\alpha \leq 1$ ) with allowing discontinuities along curves of finite length. 
As we will see later, the restriction $\alpha \leq 1$ is due to the application of the adaptive Haar wavelet basis employed for the EPWT. Note that Haar wavelets admit a straightforward transfer from one-dimensional functions along path vectors to bivariate Haar-like functions. As shown in [20], one does not necessarily need to restrict the EPWT to this simple wavelet transform. In fact, the numerical results show the enormous efficiency of the EPWT for Daubechies D4 filters and biorthogonal $7-9$ filters. These observations give rise to expect that the results presented in this paper can be extended to piecewise Hölder smooth functions of order $\alpha>1$. However, this problem would require a completely different approach since the adaptive multiresolution analysis structure obtained for Haar wavelets can not be achieved with other wavelet bases since the transfer from one-dimensional wavelet functions (along paths) to suitable bivariate functions is not longer obvious. This problem is subject of future research.

The outline of this paper is as follows. In Section 2, we first introduce the EPWT algorithm, before we apply the resulting approximation method to target functions from a function class to be described. To this end, we recall the basic ideas of the EPWT, where we show that it generates a data-dependent multiresolution analysis and a corresponding adaptive Haar wavelet basis. In order to achieve optimal approximation results, we require specific side conditions for the path vectors that are used in the EPWT algorithm. These side conditions are derived in Subsection 2.3, and further illustrations are given through a numerical example in Subsection 2.4. In Subsection 2.5, we present a strategy for the construction of path vectors which are satisfying the side conditions. Finally, Section 3 is devoted to error analysis, where we prove asymptotically optimal $N$-term error estimates of the form (1.1) for piecewise Hölder continuous functions.

\section{EPWT and Adaptive Haar Wavelet Bases}

\subsection{The EPWT Algorithm}

Suppose that $F \in L^{2}\left([0,1)^{2}\right)$ is a piecewise regular image, being uniformly regular over a finite set of regions $\left\{\Omega_{i}\right\}_{1 \leq i \leq K}$, each of whose boundaries $\partial \Omega_{i}$ is continuous and of finite length. Moreover, the set $\left\{\Omega_{i}\right\}_{1 \leq i \leq K}$ is assumed to be a disjoint partition of $[0,1)^{2}$, so that

$$
\bigcup_{i=1}^{K} \Omega_{i}=[0,1)^{2}
$$

where each closure $\bar{\Omega}_{i}$ is assumed to be a connected subset of $[0,1]^{2}$, for $i=1, \ldots, K$. Furthermore, we assume that $F$ satisfies a Hölder condition in each region $\Omega_{i}, 1 \leq i \leq K$, i.e.,

$$
|F(x)-F(x+h)| \leq C\|h\|_{2}^{\alpha}, \quad \text { for } x, x+h \in \Omega_{i}
$$

for some $\alpha \in(0,1]$ and $C>0$ which do not depend on $i$. But $F$ may be discontinuous across the boundaries between adjacent regions.

With assuming that $F$ represents a digital image, the image is given by uniform samples of $F$ over a rectangular grid. For a suitable given integer $J>1$, let $\left\{F\left(2^{-J} n\right)\right\}_{n \in I_{J}}$ be the given samples of $F$, where $I_{J}:=\left\{n=\left(n_{1}, n_{2}\right): 0 \leq n_{1} \leq 2^{J}-1,0 \leq n_{2} \leq 2^{J}-1\right\}$. We 
regard the piecewise constant function

$$
F^{2 J}(x):=\sum_{n \in I_{J}} F\left(2^{-J} n\right) \chi_{[0,1)^{2}}\left(2^{J} x-n\right) \quad \text { for } x \in[0,1)^{2}
$$

as an approximation to $F$ in $L^{2}\left([0,1)^{2}\right)$. Moreover, by

$$
\Gamma_{i}^{J}:=\left\{n \in I_{J}: \frac{n}{2^{J}} \in \Omega_{i}\right\} \quad \text { for } 1 \leq i \leq K
$$

we denote the index set of grid points that are contained in region $\Omega_{i}$, for $1 \leq i \leq K$. Obviously,

$$
\bigcup_{i=1}^{K} \Gamma_{i}^{J}=I_{J},
$$

and for the size $\# \Gamma_{i}^{J}$ of $\Gamma_{i}^{J}$ we have $\# \Gamma_{i}^{J} \leq \# I_{J}=2^{2 J}$. Consequently, (2.1) yields the error bound

$$
\left|F^{2 J}\left(2^{-J} n\right)-F^{2 J}\left(2^{-J} m\right)\right| \leq C 2^{-J \alpha}\|n-m\|_{2}^{\alpha},
$$

provided that $n, m \in \Gamma_{i}^{J}$ for some $1 \leq i \leq K$.

Remark. Note that the data $F^{2 J}$ is obtained from $F$ by interpolation, rather than by its $L^{2}$-projection onto the linear space

$$
\operatorname{span}\left\{\chi_{[0,1)^{2}}\left(2^{J} \cdot-n\right): n \in I_{J}\right\}
$$

of piecewise constant functions. We prefer to work with interpolation of $F$ in order to derive the error estimate (2.2) from the Hölder continuity of $F$ in (2.1). This way we do not need to deal with the averaging of function values for pixels lying on overlapping region boundaries.

Now let us briefly recall the EPWT algorithm from our previous work [20]. For the sake of simplicity, we use the (one-dimensional) Haar wavelet basis. To this end, let

$$
\phi_{j, k}(t):=2^{j / 2} \phi\left(2^{j} t-k\right) \quad \text { and } \quad \psi_{j, k}(t):=2^{j / 2} \psi\left(2^{j} t-k\right),
$$

with $\phi(t):=\chi_{[0,1)}(t)$ and $\psi(t):=\chi_{[0,1 / 2)}(t)-\chi_{[1 / 2,1)(t)}$, where for any interval $[a, b) \subset \mathbb{R}$, $\chi_{[a, b)}$ denotes its characteristic function.

The EPWT is a wavelet transform that works along path vectors through index subsets of $I_{J}$. For the characterization of suitable path vectors (see Subsections 2.3 and 2.5), we first need to introduce neighborhoods of indices and index sets. For any index $n=\left(n_{1}, n_{2}\right) \in$ $I_{J}$, we define its neighborhood by

$$
N(n):=\left\{m=\left(m_{1}, m_{2}\right) \in I_{J} \backslash\{n\}:\left|n_{1}-m_{1}\right| \leq 1 \text { and }\left|n_{2}-m_{2}\right| \leq 1\right\} .
$$

Hence, an interior index, i.e., an index that does not lie on the boundary of the index domain $I_{J}$, has eight neighbors. Similarly, for two disjunct index sets $I_{1} \subset I_{J}$ and $I_{2} \subset I_{J}$, we say that $I_{1}$ and $I_{2}$ are neighbors, $I_{2} \subset N\left(I_{1}\right)$, iff there exist two indices $n=\left(n_{1}, n_{2}\right) \in I_{1}$ and $m=\left(m_{1}, m_{2}\right) \in I_{2}$ such that $m \in N(n)$.

Now the EPWT algorithm is performed as follows. For the application of the first level of the EPWT we need to find a path vector $p^{2 J}=\left(p^{2 J}(n)\right)_{n=0}^{2^{2 J}-1}$ through the index set $I_{J}$. 
This path vector is a suitable permutation of all indices in $I_{J}$ and can e.g. be determined using the following strategy. Start with $p^{2 J}(0):=(0,0)$. Now, for a given $n$-th component $p^{2 J}(n)$ being contained in the index set $\Gamma_{i}^{J}$ for some $i \in\{1, \ldots, K\}$, we choose the path vectors next component $p^{2 J}(n+1)$ such that

$$
p^{2 J}(n+1) \in\left(N\left(p^{2 J}(n)\right) \cap \Gamma_{i}^{J}\right) \backslash\left\{p^{2 J}(0), \ldots, p^{2 J}(n)\right\},
$$

i.e., $p^{2 J}(n+1)$ should be a neighbor index of $p^{2 J}(n)$ in the same region set $\Gamma_{i}^{J}$ that has not been used yet in the path.

In case that $\left(N\left(p^{2 J}(n)\right) \cap \Gamma_{i}^{J}\right) \backslash\left\{p^{2 J}(0), \ldots p^{2 J}(n)\right\}$ is an empty set, the path is interrupted, and we need to start a new pathway by choosing the next index $p^{2 J}(n+1)$ from $\Gamma_{i}^{J} \backslash\left\{p^{2 J}(0), \ldots, p^{2 J}(n)\right\}$. If, however, this set is also empty, we will choose $p^{2 J}(n+1)$ from the set of remaining indices $I_{J} \backslash\left\{p^{2 J}(0), \ldots, p^{2 J}(n)\right\}$. For a more detailed description of the path vector construction we refer to [20].

In particular, for a suitably chosen path vector $p^{2 J}$, the number of interruptions can be bounded by $\tilde{K}=C_{1} K$, where $K$ is the number of regions, and where the constant $C_{1}$ does not depend on $J$, see Subsection 2.5. The obtained vector $p^{2 J}$ is composed of connected pathways, i.e., two successive components in these pathways are neighbors. We remark that in case of EPWT with Haar wavelet filters, the above strategy can even be relaxed. In fact, it suffices to choose $p^{2 J}$ such that the even path vector's components $p^{2 J}(2 n)$ and the successive odd components $p^{2 J}(2 n+1)$ are neighbors in the same index set $\Gamma_{i}^{J}$ (up to $C_{1} K$ exceptions), while $p^{2 J}(2 n+1)$ and $p^{2 J}(2 n+2)$ do not necessarily need to be neighbors.

We regard the univariate function along the path vector $p^{2 J}$

$$
\tilde{f}^{2 J}(t):=\sum_{\ell=0}^{2^{2 J}-1} F^{2 J}\left(\frac{p^{2 J}(\ell)}{2^{J}}\right) \phi\left(2^{2 J} t-\ell\right)=\sum_{\ell=0}^{2^{2 J}-1} f^{2 J}\left(p^{2 J}(\ell)\right) \phi_{2 J, \ell}(t) \quad \text { for } t \in[0,1)
$$

with $\phi_{2 J, \ell}=2^{J} \phi\left(2^{2 J} \cdot-\ell\right)$ in $(2.3)$, and $f^{2 J}\left(p^{2 J}(\ell)\right):=2^{-J} F^{2 J}\left(2^{-J} p^{2 J}(\ell)\right)$. By using (2.2), the estimate

$$
\left|\tilde{f}^{2 J}\left(2^{-2 J+1} \ell\right)-\tilde{f}^{2 J}\left(2^{-2 J}(2 \ell+1)\right)\right|=\left|F^{2 J}\left(\frac{p^{2 J}(2 \ell)}{2^{J}}\right)-F^{2 J}\left(\frac{p^{2 J}(2 \ell+1)}{2^{J}}\right)\right| \leq C 2^{(-J+1 / 2) \alpha}
$$

holds for $\ell \in\left\{0, \ldots, 2^{2 J-1}-1\right\}$, as long as $p^{2 J}(2 \ell)$ and $p^{2 J}(2 \ell+1)$ are neighbors and contained in the same index set $\Gamma_{i}^{J}$ for some $1 \leq i \leq K$. Now we apply a one-dimensional Haar wavelet transform to the given data set $\left\{F^{2 J}\left(2^{-J} n\right)\right\}_{n \in I_{J}}=\left\{\tilde{f}^{2 J}\left(2^{-2 J} \ell\right)\right\}_{\ell=0}^{2^{2 J}-1}$ along the path vector $p^{2 J}$, and so we obtain the scaling and wavelet coefficients

$$
\begin{aligned}
f^{2 J-1}(\ell):=\left\langle\tilde{f}^{2 J}, \phi_{2 J-1, \ell}\right\rangle & =2^{-J-1 / 2}\left(F^{2 J}\left(\frac{p^{2 J}(2 \ell)}{2^{J}}\right)+F^{2 J}\left(\frac{p^{2 J}(2 \ell+1)}{2^{J}}\right)\right) \\
& =2^{-1 / 2}\left(f^{2 J}\left(p^{2 J}(2 \ell)\right)+f^{2 J}\left(p^{2 J}(2 \ell+1)\right),\right. \\
g^{2 J-1}(\ell):=\left\langle\tilde{f}^{2 J}, \psi_{2 J-1, \ell}\right\rangle & =2^{-J-1 / 2}\left(F^{2 J}\left(\frac{p^{2 J}(2 \ell)}{2^{J}}\right)-F^{2 J}\left(\frac{p^{2 J}(2 \ell+1)}{2^{J}}\right)\right) \\
& =2^{-1 / 2}\left(f^{2 J}\left(p^{2 J}(2 \ell)\right)-f^{2 J}\left(p^{2 J}(2 \ell+1)\right),\right.
\end{aligned}
$$

for $\ell=0, \ldots, 2^{2 J-1}-1$, where we used the identities

$$
\begin{aligned}
\left\langle\phi\left(2^{2 J} \cdot-r\right), \phi_{2 J-1, \ell}\right\rangle & =2^{-J}\left\langle\phi_{2 J, r}, 2^{-1 / 2}\left(\phi_{2 J, 2 \ell}+\phi_{2 J, 2 \ell+1}\right)\right\rangle \\
& =2^{-J-1 / 2}\left(\delta_{r, 2 \ell}+\delta_{r, 2 \ell+1}\right)
\end{aligned}
$$


and

$$
\left\langle\phi\left(2^{2 J} \cdot-r\right), \psi_{2 J-1, \ell}\right\rangle=2^{-J-1 / 2}\left(\delta_{r, 2 \ell}-\delta_{r, 2 \ell+1}\right)
$$

with $\delta_{r, \ell}$ denoting the usual Kronecker symbol. For the wavelet coefficients, (2.4) yields the estimate

$$
\left|g^{2 J-1}(\ell)\right| \leq \frac{1}{2} C 2^{(-J+1 / 2)(\alpha+1)},
$$

if $p^{2 J}(2 \ell)$ and $p^{2 J}(2 \ell+1)$ are neighbors and contained in the same index set $\Gamma_{i}^{J}$ for some $i$.

The path vector $p^{2 J}$ determines a partition of $I_{J}$ into index sets

$$
I_{\ell}^{2 J-1}:=\left\{p^{2 J}(2 \ell), p^{2 J}(2 \ell+1)\right\} \quad \text { for } \ell=0, \ldots, 2^{2 J-1}-1 .
$$

Now we consider the "low-pass" image

$$
F^{2 J-1}(x):=\sum_{\ell=0}^{2^{2 J-1}-1} f^{2 J-1}(\ell) h_{2 J-1, \ell}(x) \quad \text { for } x \in[0,1)^{2}
$$

with the $L^{2}$-normalized characteristic functions

$$
h_{2 J-1, \ell}(x):=2^{J-1 / 2}\left(\chi_{[0,1)^{2}}\left(2^{J} x-p^{2 J}(2 \ell)\right)+\chi_{[0,1)^{2}}\left(2^{J} x-p^{2 J}(2 \ell+1)\right)\right)
$$

corresponding to the index sets $I_{\ell}^{2 J-1}$, for $\ell=0, \ldots, 2^{2 J-1}-1$. Hence, $h_{2 J-1, l}$ are normalized characteristic functions on two (usually neighboring) squares of length $2^{-J}$.

For the EPWT at the second level, where $j=2 J-1$, we first locate a second connected path vector $p^{2 J-1}=\left(p^{2 J-1}(\ell)\right)_{\ell=0}^{2^{2 J-1}-1}$ through the index sets $I_{\ell}^{2 J-1}, \ell=0, \ldots, 2^{2 J-1}-1$, i.e., the entries of $p^{2 J-1}$ form a permutation of the index set $\left\{0, \ldots, 2^{2 J-1}-1\right\}$. Similarly as before, we require that $I_{p^{2 J-1}(2 r+1)}^{2 J-1} \in N\left(I_{p^{2 J-1}(2 r)}^{2 J-1}\right)$ for all $r \in\left\{0, \ldots, 2^{2 J-2}-1\right\}$ and that $I_{p^{2 J-1}(2 r)}^{2 J-1}$ and $I_{p^{2 J-1}(2 r+1)}^{2 J-1}$ are subsets of the same index set $\Gamma_{i}^{J}$ for some $i$ (up to a finite set of indices whose size does not depend on $J$ ). Then, we apply the (one level) Haar wavelet transform to the univariate function along the path vector $p^{2 J-1}$,

$$
\tilde{f}^{2 J-1}(t):=\sum_{\ell=0}^{2^{2 J-1}-1} f^{2 J-1}\left(p^{2 J-1}(\ell)\right) \phi_{2 J-1, \ell}(t) \quad \text { for } t \in[0,1),
$$

which yields the scaling and wavelet coefficients

$$
f^{2 J-2}(\ell):=\left\langle\tilde{f}^{2 J-1}, \phi_{2 J-2, \ell}\right\rangle, \quad g^{2 J-2}(\ell):=\left\langle\tilde{f}^{2 J-1}, \psi_{2 J-2, \ell}\right\rangle, \quad \ell=0, \ldots, 2^{2 J-2}-1 .
$$

Like in the above construction, a corresponding "low pass" image

$$
F^{2 J-2}(x):=\sum_{\ell=0}^{2^{2 J-2}-1} f^{2 J-2}(\ell) h_{2 J-2, \ell}(x) \quad \text { for } x \in[0,1)^{2}
$$

is obtained, with $h_{2 J-2, \ell}$ being the $L^{2}$-normalized characteristic functions of the index sets

$$
I_{\ell}^{2 J-2}:=I_{p^{2 J-1}(2 \ell)}^{2 J-1} \cup I_{p^{2 J-1}(2 \ell+1)}^{2 J-1} \quad \text { for } \ell=0, \ldots, 2^{2 J-2}-1,
$$


i.e.,

$$
h_{2 J-2, \ell}:=2^{-1 / 2}\left(h_{2 J-1, p^{2 J-1}(2 \ell)}+h_{2 J-1, p^{2 J-1}(2 \ell+1)}\right) .
$$

We continue by iteration over the remaining levels $2 J-j$, for $j=2 J-2,2 J-3, \ldots, 0$, where at any level $2 J-j$ we first locate a path vector $p^{j+1}=\left(p^{j+1}(\ell)\right)_{\ell=0}^{2^{j+1}-1}$ through the index sets $I_{\ell}^{j+1}:=I_{p^{j+2}(2 \ell)}^{j+2} \cup I_{p^{j+2}(2 \ell+1)}^{j+2}, \ell=0, \ldots, 2^{j+1}-1$ with similar strategies as above, before the Haar wavelet transform is applied to

$$
\tilde{f}^{j+1}(t):=\sum_{\ell=0}^{2^{j+1}-1} f^{j+1}\left(p^{j+1}(\ell)\right) \phi_{j+1, \ell}(t)
$$

yielding

$$
f^{j}(\ell):=\left\langle\tilde{f}^{j+1}, \phi_{j, \ell}\right\rangle, \quad \text { and } \quad g^{j}(\ell):=\left\langle\tilde{f}^{j+1}, \psi_{j, \ell}\right\rangle, \quad \text { for } \ell=0, \ldots, 2^{j}-1 .
$$

Observe that for $j=2 J, \ldots, 0$ the index sets $I_{\ell}^{j}, \ell=0, \ldots, 2^{j}-1$ form a disjoint dyadic partition of $I_{J}$, where $\# I_{\ell}^{j}=2^{2 J-j}$. Further note that the components of the path vector $p^{2 J}$ lie in $I_{J}$, and so $p^{2 J}$ contains 2 d integer entries, whereas the path vectors $p^{j}$, with $j \leq 2 J-1$, contain $1 \mathrm{~d}$ integer entries. This is in contrast to the notation in [20].

\subsection{Adaptive Haar Wavelet Bases}

The EPWT algorithm of the previous subsection can be viewed as a tool for adaptive multiresolution analysis. We can further explain this as follows. Let us consider the space $V_{2 J}$ of piecewise constant functions

$$
V_{2 J}=\operatorname{span}\left\{h_{2 J, \ell}: \ell=0, \ldots, 2^{2 J}-1\right\},
$$

where each $h_{2 J, \ell}$ is the $L^{2}$-normalized characteristic function on one square with edge length $2^{-J}$, i.e.,

$$
h_{2 J, \ell}:=2^{J} \chi_{[0,1)^{2}}\left(2^{J} \cdot-p^{2 J}(\ell)\right) \quad \text { for } \ell=0, \ldots, 2^{2 J}-1 .
$$

Obviously, the function set $\left\{h_{2 J, \ell}: \ell=0, \ldots, 2^{2 J}-1\right\}$ forms an orthonormal basis of $V_{2 J}$, and the function $F^{2 J}$, as defined in the last subsection, can be written as

$$
F^{2 J}(x)=\sum_{\ell=0}^{2^{2 J}-1} 2^{-J} F\left(2^{-J} p^{2 J}(\ell)\right) h_{2 J, \ell}(x)=\sum_{\ell=0}^{2^{2 J}-1} f^{2 J}\left(p^{2 J}(\ell)\right) h_{2 J, \ell}(x) \quad \text { for } x \in[0,1)^{2}
$$

with $f^{2 J}\left(p^{2 J}(\ell)\right):=2^{-J} F\left(2^{-J} p^{2 J}(\ell)\right)$. By applying the first level of the EPWT with Haar filters along the path vector $\left(p^{2 J}(\ell)\right)_{\ell=0}^{2^{2 J}-1}$, we determine the coarser function spaces

$$
\begin{aligned}
V_{2 J-1}(F) & :=\operatorname{span}\left\{h_{2 J-1, \ell}:=2^{-1 / 2}\left(h_{2 J, 2 \ell}+h_{2 J, 2 \ell+1}\right) \text { for } \ell=0, \ldots, 2^{2 J-1}-1\right\} \\
W_{2 J-1}(F) & :=\operatorname{span}\left\{\Psi_{2 J-1, \ell}:=2^{-1 / 2}\left(h_{2 J, 2 \ell}-h_{2 J, 2 \ell+1}\right) \text { for } \ell=0, \ldots, 2^{2 J-1}-1\right\} .
\end{aligned}
$$


Note that the space $V_{2 J-1}(F)$ (resp. $W_{2 J-1}(F)$ ) is generated by piecewise constant functions whose support usually consists of two neighboring squares of edge length $2^{-J}$. We have $V_{2 J-1}(F) \subset V_{2 J}$ and $W_{2 J-1}(F) \subset V_{2 J}$. Moreover,

$$
V_{2 J-1}(F)+W_{2 J-1}(F)=V_{2 J}, \quad V_{2 J-1}(F) \perp W_{2 J-1}(F) .
$$

Hence, the first step of the EPWT yields an orthonormal decomposition of $F^{2 J}$ into $F^{2 J-1} \in V_{2 J-1}(F)$ and $G^{2 J-1} \in W_{2 J-1}(F)$, with $F^{2 J-1}$ in (2.6), and

$$
G^{2 J-1}(x)=\sum_{\ell=0}^{2^{2 J-1}-1} g^{2 J-1}(\ell) \Psi_{2 J-1, \ell}(x) \quad \text { for } x \in[0,1)^{2} .
$$

Proceeding further along these lines, we obtain for $j=2 J-1,2 J-2, \ldots, 0$ the adaptive scaling and wavelet spaces from the path vectors $\left(p^{j+1}(\ell)\right)_{\ell=0}^{2^{j+1}-1}$,

$$
\begin{aligned}
V_{j}(F) & :=\operatorname{span}\left\{h_{j, \ell}:=2^{-1 / 2}\left(h_{j+1, p^{j+1}(2 \ell)}+h_{j+1, p^{j+1}(2 \ell+1)}\right) \text { for } \ell=0, \ldots, 2^{j}-1\right\}, \\
W_{j}(F) & :=\operatorname{span}\left\{\Psi_{j, \ell}:=2^{-1 / 2}\left(h_{j+1, p^{j+1}(2 \ell)}-h_{j+1, p^{j+1}(2 \ell+1)}\right) \text { for } \ell=0, \ldots, 2^{j}-1\right\} .
\end{aligned}
$$

In particular, $h_{0,0}=\chi_{[0,1)^{2}}$ and $V_{0}(F)=\operatorname{span}\left\{h_{0,0}\right\}$. The support of the orthogonal basis functions $h_{j, \ell} \in V_{j}(F)$ (resp. $\Psi_{j, \ell} \in W_{j}(F)$ ) usually consists of connected areas generated by $2^{2 J-j}$ squares with edge length $2^{-J}$. Obviously, we have $V_{j}(F)+W_{j}(F)=$ $V_{j+1}(F), V_{j}(F) \perp W_{j}(F)$, and the function set

$$
\left\{h_{0,0}\right\} \cup\left\{\Psi_{j, \ell}: j=0, \ldots, 2 J-1, \ell=0, \ldots, 2^{j}-1\right\}
$$

forms an orthonormal basis of $V_{2 J}$.

The application of $2 J$ levels to a function $F^{2 J} \in V_{2 J}$ by using the EPWT algorithm finally yields a unique orthonormal decomposition

$$
F^{2 J}=F^{0}+\sum_{j=0}^{2 J-1} G^{j}
$$

with $F^{0}=f^{0}(0) h_{0,0}=f^{0}(0) \chi_{[0,1)^{2}}$, where

$$
f^{0}(0)=\int_{[0,1)^{2}} F^{2 J}(x) d x=2^{-2 J} \sum_{\ell=0}^{2^{2 J}-1} F^{2 J}\left(p^{2 J}(\ell)\right)
$$

and

$$
G^{j}(x):=\sum_{\ell=0}^{2^{j}-1} g^{j}(\ell) \Psi_{j, \ell}(x) \quad \text { for } x \in[0,1)^{2} \quad \text { for } j=0, \ldots, 2 J-1
$$

\subsection{Conditions for the Path Vectors}

In this subsection, we fix two specific side conditions for the path vectors that are required for our error analysis in the following Section 3. The two side conditions are termed (a) region condition and (b) diameter condition, as stated below. 
To introduce the two conditions, let $J>0$ be an arbitrary fixed integer. Suppose that the path vectors $\left(p^{j}(\ell)\right)_{\ell=0}^{2^{j}-1}, j=1, \ldots, 2 J$, are iteratively chosen at the levels of the EPWT algorithm. We assume that their corresponding index sets $I_{\ell}^{j}, \ell=0, \ldots, 2^{j}-1$,

$$
\begin{aligned}
I_{\ell}^{2 J} & :=\left\{p^{2 J}(\ell)\right\} \quad \text { for } \ell=0, \ldots, 2^{2 J}-1, \\
I_{\ell}^{j} & :=I_{p^{j+1}(2 \ell)}^{j+1} \cup I_{p^{j+1}(2 \ell+1)}^{j+1} \quad \text { for } \ell=0, \ldots, 2^{j}-1,1 \leq j<2 J,
\end{aligned}
$$

are satisfying the following two conditions for every $J>0$.

The region condition ensures that at each level of the EPWT the path vector is chosen in a way such that all indices belonging to one region index set $\Gamma_{i}, i=1, \ldots, K$, are taken first before crossing over to the next region. In this way, only a small number of index sets $I_{\ell}^{j}$ containing indices from different regions arises.

The diameter condition ensures that the diameter of the constructed index sets $I_{\ell}^{j}$ does not grow too fast but only by a factor $\sqrt{2}$ going from one level to the next. That means, one should avoid long thin index sets. Let us determine the two conditions more precisely.

(a) Region condition. After the performance of the $(2 J-j)$ th level of the EPWT, $j \in\{2 J-1, \ldots, 0\}$, there are at most $C_{1} K$ index sets $I_{\ell}^{j}$, whose components are not completely contained in one region index set $\Gamma_{i}^{J}$ for some $i \in\{1, \ldots, K\}$, and the constant $C_{1}$ does not depend on $J$ or $j$.

(b) Diameter condition. After the performance of the $(2 J-j)$ th level of the EPWT, $j \in\{2 J-1, \ldots, 0\}$, almost all index sets $I_{\ell}^{j}, \ell \in\left\{0, \ldots, 2^{j}-1\right\}$, whose components are completely contained in one region index set $\Gamma_{i}^{J}$, for some $i \in\{1, \ldots, K\}$, possess an almost optimal "diameter", i.e., there is a constant $D<\infty$, being independent of $J$ and $j$, such that

$$
\operatorname{diam} I_{\ell}^{j}=\max _{k_{1}, k_{2} \in I_{\ell}^{j}}\left\|k_{1}-k_{2}\right\|_{2} \leq D 2^{J-j / 2} .
$$

Moreover, the number of index sets with $I_{\ell}^{j} \subset \Gamma_{i}^{J}$, for some $i$, that do not satisfy this optimal diameter condition, is bounded by a constant $C_{2}<\infty$ which does not depend on $J$ or $j$.

For notational simplicity, we denote by $\Lambda^{j}$ the set of all indices $\ell \in\left\{0, \ldots, 2^{j}-1\right\}$ for which the index set $I_{\ell}^{j}$ is contained in $\Gamma_{i}^{J}$, for some $i$, and which satisfies the diameter condition (2.7). By assumptions (a) and (b), at each level $2 J-j$, the number of indices $\ell$ being not contained in $\Lambda^{j}$ is bounded by $C_{1} K+C_{2}$.

Remark. Since each index set $I_{\ell}^{j}$ has by definition $2^{2 J-j}$ elements, the diameter condition can for instance be satisfied for even $j$, if the indices in $I_{\ell}^{j}$ are arranged in a square of length $2^{J-j / 2}$. In this case, condition $(2.7)$ holds with $D=\sqrt{2}$.

\subsection{Example}

For the purpose of illustration, we consider one specific example for an image of size $16 \times 16$. This numerical example demonstrates the efficacy of the EPWT algorithm. Furthermore, it helps to explain the region condition and the diameter condition of the 
previous subsection. To this end, we consider a piecewise Hölder continuous function $F$ and its interpolation $F^{2 J}$ for $J=4$, see Figure 1(a). The image $F^{2 J}$ consists of three regions, and we assume that the Hölder condition (2.2) (with a suitable constant $C$ ) is satisfied in each of these regions.

We aim to apply the EPWT algorithm such that the region condition (a) and the diameter condition (b) are satisfied with small constants $C_{1}, D$, and $C_{2}$. In a first step, we determine a path $p^{8}$ of length 256 that is built by concatenating three connected pathways $p_{1}^{8}$ (background), $p_{2}^{8}$ (approximation of circle) and $p_{3}^{8}$ (approximation of sloping bar), see Figure 1(b). The first indices of the pathways $p_{1}^{8}, p_{2}^{8}$ and $p_{3}^{8}$ are indicated by small circles. In this example, the vectors $p_{\nu}^{8}$, for $\nu=1,2,3$, are even completely connected, i.e., we have $p_{\nu}^{8}(\ell+1) \in N\left(p_{\nu}^{8}(\ell)\right)$, for $\nu=1,2,3$ and for $\ell=0, \ldots, \# \Gamma_{\nu}^{4}-2$. The path vector $p^{8}$ determines a low-pass image $F^{7}$, where we have also indicated the index sets $I_{\ell}^{7}, \ell=0, \ldots, 127$ that are determined by $p^{8}$, see Figure $1(\mathrm{c})$.

There is only one index set, which does not satisfy the region condition, see the last index $(0,15)$ of $p_{1}^{8}$ in the right upper corner and the first index $(2,4)$ of $p_{2}^{8}$ in Figure $1(\mathrm{c})$. Hence, we have $C_{1}=1 / 3$. Since there are index sets $I_{\ell}^{7}$ of the form $\{m, n\}$ with $\|m-n\|_{2}=$ $\sqrt{2}$, the diameter condition (2.7) is satisfied with $D=1$, and we have $C_{2}=0$.

At the second level of the EPWT, a suitable path vector $p^{7}$ determines a low-pass image $F^{6}$, see Figure 1(d), with indicated index sets of size 4 . There are only two index sets, which do not satisfy the region condition, i.e., $C_{1}=2 / 3$. Furthermore, there are two index sets at the bottom of the image, which satisfy the diameter condition (2.7) only with the constant $D=3 / 2$, wheras all other index sets satisfy (2.7) with $D \leq \sqrt{5} / 2$.

At the third level of the EPWT, a path vector $p^{6}$ determines the low-pass image $F^{5}$, see Figure 1(e), where we have also indicated the index sets of size 8. An appropriate concatenation of those two index sets of size 4 that did not meet the region condition in $F^{6}$ now leads to only one exception of the region condition, i.e., $C_{1}=1 / 3$. We observe that all other index sets in Figure 1(e) satisfy the diameter condition $(2.7)$ with $D=$ $\sqrt{18} / \sqrt{8}=3 / 2$.

At the fourth level of the EPWT, we obtain the low-pass image $F^{4}$ in Figure 1(f) with only one index set violating the region condition. Five index sets satisfy the diameter condition with $D=\sqrt{45} / 4 \approx 1.67705$. The remaining index sets satisfy (2.7) with $D \leq$ $\sqrt{40} / 4 \approx 1.58114$. In this case, (level-independent) constants are $C_{1}=2 / 3, D=\sqrt{45} / 4$, and $C_{2}=0$.

\subsection{Algorithm for the Path Vector Construction}

Now let us turn to the construction of the path vectors. Recall that the path vectors are required to satisfy the region condition and diameter condition in Subsection 2.3. In fact, these two conditions can easily be satisfied by suitable path vector construction. They are already implicitly encoded in the relaxed EPWT algorithm introduced in [20]. There, the path $p^{j}$ is constructed by fixing a next path component $p^{j}(\ell+1)$ such that the corresponding function values $f\left(p^{j}(\ell)\right)$ and $f\left(p^{j}(\ell+1)\right)$ differ not too much, i.e., such that $\mid f\left(\left(p^{j}(\ell)\right)-f\left(p^{j}(\ell+1)\right) \mid\right.$ is smaller than a predetermined bound $\theta$. This procedure ensures the region condition since this comparison of neighboring function values works like an implicit edge detector. Further, the diameter condition is encoded in the path construction using the so called "Center Hybrid" strategy proposed in [22]. Here, one 
(a)

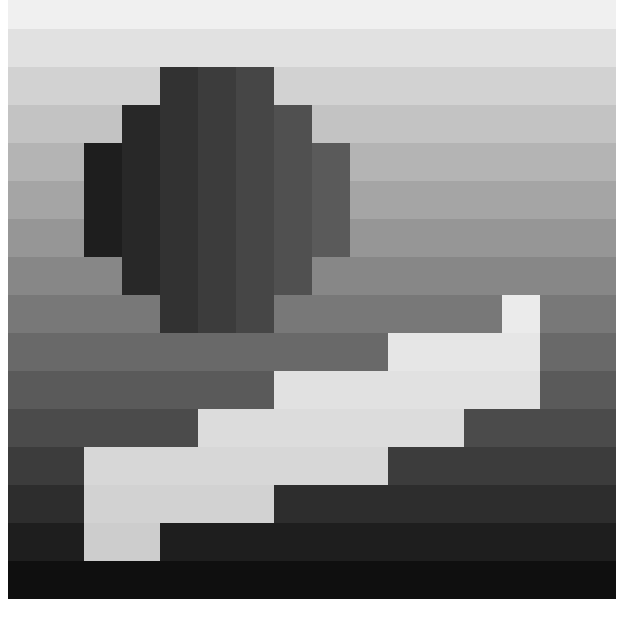

(b)

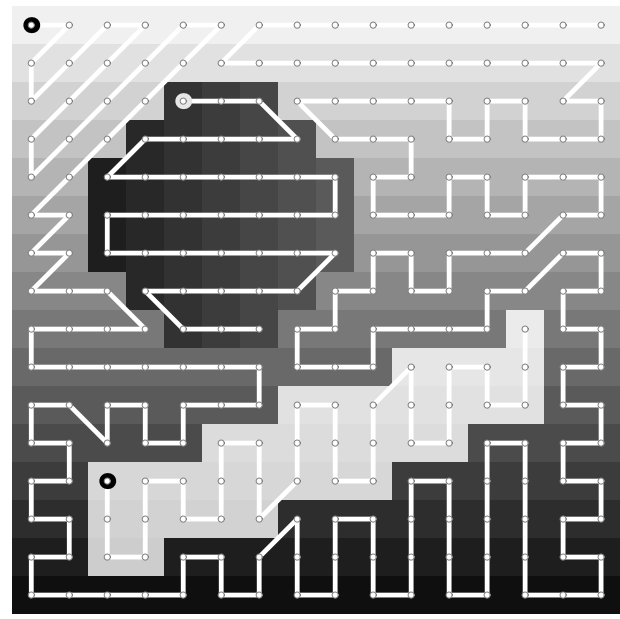

(c)

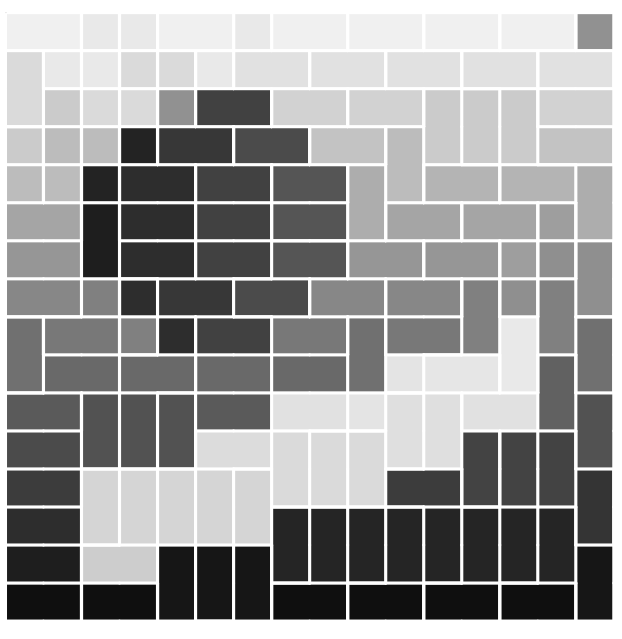

(d)

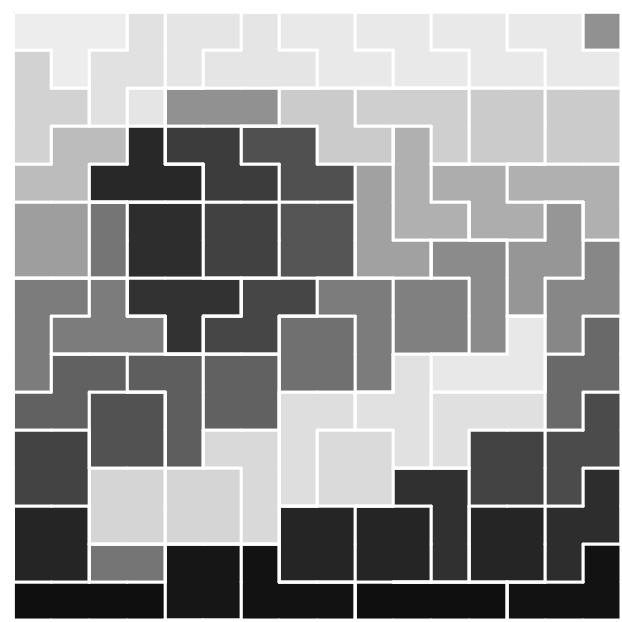

(f)

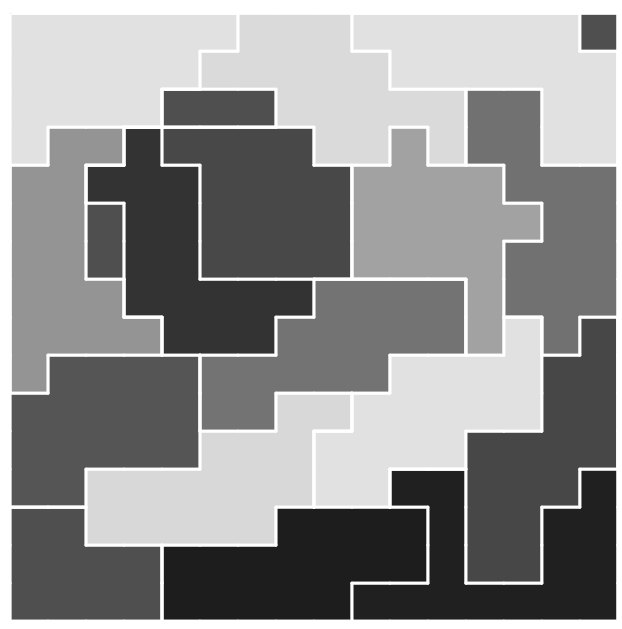

(e)

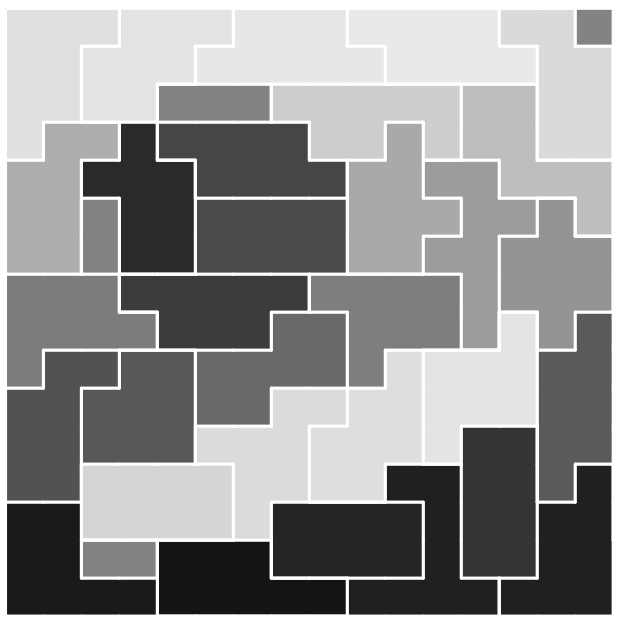

Figure 1: Application of the EPWT algorithm to a $16 \times 16$ image. (a) interpolated image $F^{8}$, (b) connected path $p^{8}$ for $F^{8}$, (c) low-pass image $F^{7}$ with index sets of size 2, (d) low-pass image $F^{6}$ with index sets of size 4 , (e) low-pass image $F^{5}$ with index sets of size 8, (f) low-pass image $F^{4}$ with index sets of size 16. 
computes the centers (mean values) of the index sets $I_{\ell}^{j}$ and chooses the path vectors next component such that the distance between centers of neighboring index sets is minimal (and provided that the corresponding function values differ by a value smaller that the bound $\theta$ ).

A slightly different idea for path construction ensuring the two path conditions is given in the following procedure. Assuming that the image consists of a finite number of smooth regions, we may apply an edge detector to the image in order to determine the regions $\Omega_{i}, i=1, \ldots, K$, and their corresponding index sets $\Gamma_{i}^{J}$. At level $(2 J-j+1)$, the construction of the path vectors $p^{j}=\left(p^{j}(\ell)\right)_{\ell=0}^{2^{j}-1}$ is, through their index sets $I_{\ell}^{j}$, performed by the application of the following algorithm.

\section{Algorithm}

Let $i=1, \ell=0$.

1. Choose an index set $p^{j}(2 \ell) \in \Gamma_{i}^{J} \backslash\left\{p^{j}(0), \ldots, p^{j}(2 \ell-1)\right\}$ possessing the smallest positive number of neighbor index sets in $\Gamma_{i}^{J}$ and go to 2 . If such an index set does not exist, and if $i<K$, let $i=i+1$ and go to 1 . Otherwise, go to 3 .

2. Choose $p^{j}(2 \ell+1)$ such that

$$
I_{p^{J}(2 \ell+1)}=\underset{I_{k}^{j} \in \Gamma_{i}^{J} \backslash\left\{p^{j}(0), \ldots, p^{j}(2 \ell)\right\}}{\operatorname{argmin}}\left\{\operatorname{diam}\left(I_{p^{j}(2 \ell)}^{j} \cup I_{k}^{j}\right)\right\} .
$$

Let $\ell=\ell+1$ and go to 1 .

3. Take all index sets that can not be incorporated into the path using the two steps above to built the remaining pairs $p^{j}(2 \ell)$ and $p^{j}(2 \ell+1)$ leading to new index sets $I_{\ell}^{j-1}=I_{p^{j}(2 \ell)}^{j} \cup I_{p^{j}(2 \ell+1)}^{j}$.

\section{Approximation Properties of the EPWT Algorithm}

Recall that for given integer $J>0$, the function $F^{2 J}$ is assumed to be the piecewise constant approximation of the image $F$ satisfying (2.2). In this section, we shall prove the optimal $N$-term approximation to $F$ by a suitably chosen EPWT, where the path vectors are required to satisfy the region condition and the diameter condition of Subsection 2.3.

Let us first prove suitable estimates for the scaling and the wavelet coefficients.

Theorem 3.1 Let $F^{2 J}$ be an image in $V^{2 J}$ satisfying (2.2) for each index set $\Gamma_{i}^{J}, i=$ $1, \ldots, K$, as determined in Subsection 2.1. For the application of the EPWT to $F^{2 J}$ (according to Section 2), we assume that the path vectors $\left(p^{j+1}(\ell)\right)_{\ell=0}^{2^{j+1}-1}, j=2 J-1, \ldots, 0$, satisfy the region condition (a) and the diameter condition (b) of Subsection 2.3. Let $f^{2 J}\left(p^{2 J}(\ell)\right):=2^{-J} F^{2 J}\left(2^{-J} p^{2 J}(\ell)\right), \ell=0, \ldots, 2^{2 J}-1$, and let $f^{j}(\ell)=\left\langle\tilde{f}^{j+1}, \phi_{j, \ell}\right\rangle, j=$ $2 J-1,2 J-2, \ldots, 0, \ell=0, \ldots, 2^{j}-1$ be the scaling coefficients that are obtained by the $E P W T$. Then, for all $j=2 J, \ldots, 0$ and $\ell \in \Lambda^{j-1}$, the estimate

$$
\left|f^{j}\left(p^{j}(2 \ell)\right)-f^{j}\left(p^{j}(2 \ell+1)\right)\right| \leq 2^{\alpha / 2} C D^{\alpha} 2^{-j(\alpha+1) / 2}
$$


holds, where $D>1$ is the constant of the diameter condition (2.7), and where $C$ and $\alpha$ are the Hölder constant and the Hölder exponent in (2.2). Furthermore, for all $\ell \in$ $\left\{0, \ldots, 2^{j-1}-1\right\} \backslash \Lambda^{j-1}$, we find the estimate

$$
\left|f^{j}\left(p^{j}(2 \ell)\right)-f^{j}\left(p^{j}(2 \ell+1)\right)\right| \leq C^{\prime} 2^{-j / 2}
$$

with some constant $C^{\prime}$ being independent of $J$ and $j$.

Proof. For $j=2 J$, estimate (3.1) follows directly from (2.4). By using the representation $\phi_{2 J-1, \ell}=2^{-1 / 2}\left(\phi_{2 J, 2 \ell}+\phi_{2 J, 2 \ell+1}\right)$ we find

$$
f^{2 J-1}(\ell)=2^{-J-1 / 2}\left(F^{2 J}\left(\frac{p^{2 J}(2 \ell)}{2^{J}}\right)+F^{2 J}\left(\frac{p^{2 J}(2 \ell+1)}{2^{J}}\right)\right)=2^{-J-1 / 2} \sum_{n \in I_{\ell}^{2 J-1}} F^{2 J}\left(\frac{n}{2^{J}}\right),
$$

see (2.5). Hence,

$$
\begin{aligned}
& \left|f^{2 J-1}\left(p^{2 J-1}(2 \ell)\right)-f^{2 J-1}\left(p^{2 J-1}(2 \ell+1)\right)\right| \\
& \quad=2^{-J-1 / 2}\left|\sum_{n \in I_{p^{2 J-1}(2 \ell)}^{2 J-1}} F^{2 J}\left(\frac{n}{2^{J}}\right)-\sum_{n \in I_{p^{2 J-1}(2 \ell+1)}^{2 J-1}} F^{2 J}\left(\frac{n}{2^{J}}\right)\right| \\
& \quad \leq 2^{-J-1 / 2} 2 C\left(\frac{2 D}{2^{J}}\right)^{\alpha}=2^{\alpha / 2} C D^{\alpha} 2^{-(J-1 / 2)(\alpha+1)},
\end{aligned}
$$

follows by $I_{\ell}^{2 J-2}=I_{p^{2 J-1}(2 \ell)}^{2 J-1} \cup I_{p^{2 J-1}(2 \ell+1)}^{2 J-1}$ and (2.2), where we note that the sets $I_{p^{2 J-1}(2 \ell)}^{2 J-1}$ and $I_{p^{2 J-1}(2 \ell+1)}^{2 J-1}$ contain only two indices and, moreover, the diameter condition

$$
\operatorname{diam} I_{\ell}^{2 J-2} \leq 2 D
$$

holds. Likewise, for general $j \in\{1, \ldots, 2 J-1\}$ we observe that $f^{j}(\ell)$, as obtained by the application of the $(2 J-j)$ th level of the EPWT algorithm, can be viewed as a weighted average of function values $F^{2 J}\left(2^{-J} n\right)$ with $n \in I_{\ell}^{j}$, i.e.,

$$
f^{j}(\ell)=2^{-J} \frac{1}{2^{J-j / 2}} \sum_{n \in I_{\ell}^{j}} F^{2 J}\left(\frac{n}{2^{J}}\right),
$$

where $\# I_{\ell}^{j}=2^{2 J-j}$. Hence, by using the diameter property for $\ell \in \Lambda^{j-1}$, we obtain

$$
\begin{aligned}
\left|f^{j}\left(p^{j}(2 \ell)\right)-f^{j}\left(p^{j}(2 \ell+1)\right)\right| & =2^{-2 J+j / 2}\left|\sum_{n \in I_{p^{j}(2 \ell)}^{j}} F^{2 J}\left(\frac{n}{2^{J}}\right)-\sum_{n \in I_{p^{j}(2 \ell+1)}} F^{2 J}\left(\frac{n}{2^{J}}\right)\right| \\
& \leq 2^{-2 J+j / 2} 2^{2 J-j} C\left(\frac{D 2^{J-(j-1) / 2}}{2^{J}}\right)^{\alpha} \\
& =2^{\alpha / 2} C D^{\alpha} 2^{-j(\alpha+1) / 2} .
\end{aligned}
$$

Finally, since $F$ (resp. $F^{2 J}$ ) is bounded, we obtain

$$
\left|f^{j}\left(p^{j}(2 \ell)\right)-f^{j}\left(p^{j}(2 \ell+1)\right)\right| \leq C^{\prime} 2^{-j / 2}
$$

for all indices $\ell \in\left\{0, \ldots, 2^{j-1}-1\right\} \backslash \Lambda^{j-1}$, with some constant $C^{\prime}$ being independent from $j$. Note that the last estimate follows from the previous one by letting $\alpha=0$.

We are now in a position to estimate the wavelet coefficients obtained by the EPWT. 
Theorem 3.2 For $j=2 J-1, \ldots, 0$, let $g^{j}(\ell)=\left\langle\tilde{f}^{j+1}, \psi_{j, \ell}\right\rangle, \ell=0, \ldots, 2^{j}-1$, denote the wavelet coefficients that are obtained by applying the EPWT algorithm to $F^{2 J} \in V_{2 J}$ (according to Section 2), where we assume that $F^{2 J}$ satisfies (2.2). Further assume that the path vectors $\left(p^{j+1}(\ell)\right)_{\ell=0}^{2^{j+1}-1}, j=2 J-1, \ldots, 0$, in the EPWT algorithm satisfy the region condition (a) and the diameter condition (b) of Subsection 2.3. Then, for all $j=$ $2 J-1, \ldots, 0$ and $\ell \in \Lambda^{j}$, the estimate

$$
\left|g^{j}(\ell)\right| \leq \frac{1}{2} C D^{\alpha} 2^{-j(\alpha+1) / 2}
$$

holds, where $D>1$ is the constant of the diameter condition (2.7), and where $C$ and $\alpha$ are the Hölder constant and the Hölder exponent in (2.2). Furthermore, for all $\ell \in$ $\left\{0, \ldots, 2^{j}-1\right\} \backslash \Lambda^{j}$, we find the estimate

$$
\left.\mid g^{j}(\ell)\right) \mid \leq \frac{1}{2} C^{\prime} 2^{-j / 2}
$$

with some constant $C^{\prime}$ being independent of $J$ and $j$.

Proof. The proof follows from Theorem 3.1, with observing that the one-dimensional Haar wavelet satisfies $\psi_{j, \ell}=2^{-1 / 2}\left(\phi_{j+1,2 \ell}-\phi_{j+1,2 \ell+1}\right)$, and by using $\left\langle\phi_{j+1, r}, \phi_{j+1, \ell}\right\rangle=\delta_{r, \ell}$. By (3.1), we obtain

$$
\begin{aligned}
\left|g^{j}(\ell)\right| & =\left|\left\langle\tilde{f}^{j+1}, \psi_{j, \ell}\right\rangle\right| \\
& =\left|\sum_{r=0}^{2^{j+1}-1} f^{j+1}\left(p^{j+1}(r)\right)\left\langle\phi_{j+1, r}, 2^{-1 / 2}\left(\phi_{j+1,2 \ell}-\phi_{j+1,2 \ell+1}\right)\right\rangle\right| \\
& =2^{-1 / 2}\left|f^{j+1}\left(p^{j+1}(2 \ell)\right)-f^{j+1}\left(p^{j+1}(2 \ell+1)\right)\right| \\
& \leq 2^{-1 / 2} 2^{\alpha / 2} C D^{\alpha} 2^{-(j+1)(\alpha+1) / 2}=\frac{1}{2} C D^{\alpha} 2^{-j(\alpha+1) / 2} .
\end{aligned}
$$

Likewise, for all $\ell \in\left\{0, \ldots, 2^{j}-1\right\} \backslash \Lambda^{j}$

$$
\left|g^{j}(\ell)\right| \leq 2^{-1 / 2} C^{\prime} 2^{-(j+1) / 2}=C^{\prime} 2^{-(j+2) / 2}
$$

follows from (3.2).

Observe that the complete image $F^{2 J}$ is now covered by the vector of wavelet coefficients (as generated by the EPWT)

$$
\mathbf{g}=\left(\left(g^{2 J-1}\right)^{T}, \ldots, g^{0}, g^{-1}\right)^{T}
$$

with $g^{j}=\left(g^{j}(\ell)\right)_{\ell=0}^{2^{j}-1}$ for $j=0, \ldots, 2 J-1$, and the mean value

$$
g^{-1}=g^{-1}(0):=f^{0}(0)=2^{-2 J} \sum_{n \in I_{J}} F^{2 J}\left(2^{-J} n\right),
$$

together with the side information on the path vectors in each iteration step

$$
\mathbf{p}=\left(\left(p^{2 J}\right)^{T}, \ldots,\left(p^{1}\right)^{T}\right)^{T} \in \mathbb{R}^{2\left(2^{2 J}-1\right)} .
$$


In order to find a sparse representation of the digital image $F^{2 J}$, we apply a shrinkage procedure to the EPWT wavelet coefficients $g^{j}(\ell)$, using the hard threshold function

$$
s_{\sigma}(x)= \begin{cases}x & |x| \geq \sigma \\ 0 & |x|<\sigma\end{cases}
$$

We now study the error of a sparse representation using only the $N$ wavelet coefficients with largest absolute value for an approximative reconstruction of $F^{2 J}$. For convenience, let $S_{N}^{2 J}$ be the set of indices $(j, \ell)$ of the $N$ wavelet coefficients with largest absolute value.

Using the orthogonal decomposition of $F^{2 J}$ of Subsection 2.2, the $L^{2}$-error can be represented as

$$
\epsilon_{N}=\left\|F^{2 J}-F_{N}^{2 J}\right\|_{2}^{2}=\sum_{(j, \ell) \notin S_{N}^{2 J}}\left|g^{j}(\ell)\right|^{2},
$$

where $F_{N}^{2 J}$ is the approximation of $F^{2 J}$ that is reconstructed from the $N$ wavelet coefficients $g^{j}(\ell),(j, \ell) \in S_{N}^{2 J}$ with largest absolute value.

Now we prove the main result of this paper, by showing the optimal $N$-term approximation of the EPWT algorithm.

Theorem 3.3 Let $F_{N}^{2 J}$ be the $N$-term approximation of $F^{2 J}$ as constructed above, and let the assumptions of Theorem 3.2 be satisfied. Then the estimate

$$
\epsilon_{N}=\left\|F^{2 J}-F_{N}^{2 J}\right\|_{2}^{2} \leq \tilde{C} N^{-\alpha}
$$

holds for all $J \in \mathbb{N}$, where the constant $\tilde{C}<\infty$ does not depend on $J$.

Proof. We organize the proof into two parts.

1. Let the sequence of all wavelet coefficients $g^{j}(\ell), j=0, \ldots, 2 J-1, \ell=0, \ldots, 2^{j}-1$, and $g^{-1}=g^{-1}(0)$ be sorted in decreasing order, such that we obtain the new sequence $\left(g_{\mu}\right)_{\mu=0}^{2^{2 J}-1}$ with $\left|g_{\mu}\right| \geq\left|g_{\mu+1}\right|$ for $\mu=0, \ldots, 2^{2 J}-2$.

We first show that $\left\|\left(g_{\mu}\right)_{\mu=0}^{2^{2 J}-1}\right\|_{l^{p}}^{p}=\sum_{\mu=0}^{2^{2 J}-1}\left|g_{\mu}\right|^{p}$ with $\frac{1}{2}<\frac{1}{p}<\frac{\alpha+1}{2}$ is bounded independently from the choice of the integer $J>1$. For that purpose, we use the estimates in Theorem 3.2, where we distinguish between type I wavelet coefficients $g^{j}(\ell)$ satisfying the estimate (3.3) and type II wavelet coefficients satisfying only the estimate (3.4). From the region condition (a) and the diameter condition (b) on the path vectors $p^{j}$, it follows that there are at most $C_{1} K+C_{2}$ wavelet coefficients of type II in each level $j$ and the sum of these type II coefficients is bounded by

$$
\begin{aligned}
\sum_{g_{\mu} \text { of type II }}\left|g_{\mu}\right|^{p} & \leq\left|g^{-1}(0)\right|^{p}+\left(C_{1} K+C_{2}\right) \sum_{j=0}^{2 J-1}\left(\frac{1}{2} C^{\prime} 2^{-j / 2}\right)^{p} \\
& =\left|g^{-1}(0)\right|^{p}+\left(C_{1} K+C_{2}\right)\left(C^{\prime} / 2\right)^{p} \sum_{j=0}^{2 J-1} 2^{-j p / 2} \\
& \leq\left|g^{-1}(0)\right|^{p}+\left(C_{1} K+C_{2}\right)\left(C^{\prime} / 2\right)^{p}\left(1-2^{-p / 2}\right)^{-1}
\end{aligned}
$$

for all $p>0$, independently from $J$. 
For the type I coefficients we obtain the bound

$$
\begin{aligned}
\sum_{g_{\mu} \text { of type I }}\left|g_{\mu}\right|^{p} & \leq \sum_{j=0}^{2 J-1} 2^{j}\left|\left(C D^{\alpha} / 2\right) 2^{-j(\alpha+1) / 2}\right|^{p} \\
& =\left(C D^{\alpha} / 2\right)^{p} \sum_{j=0}^{2 J-1} 2^{-j(p(\alpha+1) / 2-1)}
\end{aligned}
$$

by using (3.3). This expansion is finite (independent from $J$ ), if $p(\alpha+1) / 2>1$, i.e., if $\frac{1}{p}<\frac{\alpha+1}{2}$.

2. We apply now the following result by Stechkin (see e.g. [9]):

Let $\mathcal{F}$ be a finite or countable index set, $0<p \leq q$, and $\alpha=\left(\alpha_{\mu}\right)_{\mu \in \mathcal{F}}$ be a sequence in $l^{p}(\mathcal{F})$. If $\mathcal{F}_{N}$ is the set of indices corresponding to the $N$ largest values of $\left|\alpha_{\mu}\right|$, we have

$$
\sum_{\mu \notin \mathcal{F}_{N}}\left|\alpha_{\mu}\right|^{q} \leq\|\alpha\|_{l^{p}}^{q} N^{-r q}
$$

where $r:=\frac{1}{p}-\frac{1}{q} \geq 0$.

In our case, we choose $\mathcal{F}=\left\{0, \ldots, 2^{2 J}-1\right\}, q=2$ and $p$ as before. Then we obtain from (3.7) with $r q=2 r=\frac{2}{p}-1$ for the considered sequence $\left(g_{\mu}\right)_{\mu=0}^{2^{2 J}-1}$ the inequality

$$
\sum_{\mu=N+1}^{2^{2 J}-1}\left|g_{\mu}\right|^{2} \leq\left\|\left(g_{\mu}\right)_{\mu=0}^{2^{2 J}-1}\right\|_{l^{p}}^{2} N^{1-2 / p} .
$$

For $p \rightarrow 2 /(\alpha+1)$ the assertion of the theorem follows.

Let us conclude by stating the following corollary.

Corollary 3.4 Let $F \in L^{2}\left([0,1)^{2}\right)$ be piecewise Hölder continuous (as assumed in Subsection 2.1). Then, for any $\epsilon>0$ there exists an integer $J(\epsilon)$, such that for all $J \geq J(\epsilon)$ the $N$-term estimate

$$
\left\|F-F_{N}^{2 J}\right\|_{L^{2}}^{2}<\tilde{C} N^{-\alpha}+\epsilon
$$

holds, where $\tilde{C}$ is the constant in (3.6).

Proof. For given $J>0$ and $n=\left(n_{1}, n_{2}\right) \in I_{J}$ let

$$
A_{n}^{J}:=\left[2^{-J} n_{1}, 2^{-J}\left(n_{1}+1\right)\right) \times\left[2^{-J} n_{2}, 2^{-J}\left(n_{2}+1\right)\right) .
$$

Then,

$$
\left\|F-F^{2 J}\right\|_{L^{2}}^{2}=\sum_{n \in I_{J}} \int_{A_{n}^{J}}\left|F(x)-F\left(2^{-J} n\right)\right|^{2} d x .
$$

We consider two types of indices $n \in I_{J}$. If $A_{n}^{J} \cap \partial \Omega_{i}=\emptyset$ for all $i \in\{1, \ldots, K\}$, we say that $n$ belongs to the subset $I_{J}^{\prime}$ of $I_{J}$. Otherwise, it belongs to $I_{J}^{\prime \prime}$. With assuming finite length for $\cup_{i=1}^{K} \partial \Omega_{i}$, there exists a constant $L$ being independent of $J$, such that \# $I_{J}^{\prime \prime} \leq L 2^{J}$ for all $J$. For the indices in $I_{J}^{\prime}$ we can use the Hölder condition (2.1) and find

$$
\sum_{n \in I_{J}^{\prime}} \int_{A_{n}^{J}}\left|F(x)-F\left(2^{-J} n\right)\right|^{2} d x \leq \sum_{n \in I_{J}^{\prime}} 2^{-2 J}\left(C 2^{(-J+1 / 2) \alpha}\right)^{2} \leq C^{2} 2^{(-2 J+1) \alpha}
$$


with using $\# I_{J}^{\prime} \leq \# I_{J}=2^{2 J}$. Since the image $F$ is bounded, i.e., $|F(x)|<C^{\prime}$ for some $C^{\prime}>0$, we also have

$$
\sum_{n \in I_{J}^{\prime \prime}} \int_{A_{n}^{J}}\left|F(x)-F\left(2^{-J} n\right)\right|^{2} d x<\sum_{n \in I_{J}^{\prime \prime}} 2^{-2 J}\left(2 C^{\prime}\right)^{2}<2^{-J+2} L C^{\prime} .
$$

Hence, $\left\|F-F^{2 J}\right\|_{L^{2}}^{2}<C^{2} 2^{(-2 J+1) \alpha}+2^{-J+2} L C^{\prime}$ holds. Moreover, for any $\epsilon>0$ we can find an integer $J(\epsilon)$, such that $\left\|F-F^{2 J}\right\|_{L^{2}}^{2}<\epsilon$ holds for all $J \geq J(\epsilon)$. This in combination with (3.6) concludes our proof.

\section{Acknowledgment}

This work is supported by the priority program SPP 1324 of the Deutsche Forschungsgemeinschaft (DFG), projects PL 170/13-1 and IS 58/1-1.

\section{References}

[1] F. Arandiga, A. Cohen, R. Donat, N. Dyn, and B. Matei, Approximation of piecewise smooth functions and images by edge-adapted (ENO-EA) nonlinear multiresolution techniques, Appl. Comput. Harmon. Anal. 24 (2008), 225-250.

[2] E.J. Candès and D.L. Donoho, New tight frames of curvelets and optimal representations of objects with piecewise singularities, Comm. Pure Appl. Math. 57 (2004), 219-266.

[3] E.J. Candès, L. Demanet, D.L. Donoho, and L. Ying, Fast discrete curvelet transforms, Multiscale Model. Simul. 5 (2006), 861-899.

[4] A. Cohen, W. Dahmen, I. Daubechies, and R.A. DeVore, Harmonic analysis of the space BV, Rev. Mat. Iberoam. 19(1) (2003), 235-263.

[5] A. Cohen, R.A. DeVore, P. Petrushev, and H. Xu, Nonlinear approximation and the space $B V\left(\mathbb{R}^{2}\right)$, Amer. J. Math. 121 (1999), 587-628.

[6] I. Daubechies, Ten Lectures on Wavelets, SIAM, Philadelphia, 1992.

[7] S. Dekel and D. Leviatan, Adaptive multivariate approximation using binary space partitions and geometric wavelets, SIAM J. Numer. Anal. 43 (2006), 707-732.

[8] L. Demaret, N. Dyn, and A. Iske, Image compression by linear splines over adaptive triangulations, Signal Processing 86 (2006), 1604-1616.

[9] R.A. DeVore, Nonlinear approximation, Acta Numerica, 1998, 51-150.

[10] M.N. Do and M. Vetterli, The contourlet transform: an efficient directional multiresolution image representation, IEEE Trans. Image Process. 14 (2005), 2091-2106.

[11] D.L. Donoho, Wedgelets: Nearly minimax estimation of edges, Ann. Stat. 27 (1999), 859-897.

[12] K. Guo and D. Labate, Optimally sparse multidimensional representation using shearlets, SIAM J. Math. Anal. 39 (2007), 298-318.

[13] K. Guo, W.-Q. Lim, D. Labate, G. Weiss, and E. Wilson, Wavelets with composite dilations, Electr. res. Announc. of AMS 10 (2004), 78-87. 
[14] A. Harten, Multiresolution representation of data: general framework, SIAM J. Numer. Anal. 33 (1996), 1205-1256.

[15] L. Jaques and J.-P. Antoine, Multiselective pyramidal decomposition of images: wavelets with adaptive angular selectivity, Int. J. Wavelets Multiresolut. Inf. Process. 5 (2007), 785-814.

[16] B. Karaivanov and P. Petrushev, Nonlinear piecewise polynomial approximation beyond Besov spaces, Appl. Comput. Harmon. Anal. 15 (2003), 177-223.

[17] E. Le Pennec and S. Mallat, Bandelet image approximation and compression, Multiscale Model. Simul. 4 (2005), 992-1039.

[18] S. Mallat, A wavelet tour of signal processing, Academic Press, San Diego, 1999.

[19] S. Mallat, Geometrical grouplets, Appl. Comput. Harmon. Anal. 26 (2009), 161-180.

[20] G. Plonka, The easy path wavelet transform: a new adaptive wavelet transform for sparse representation of two-dimensional data, Multiscale Modelling Simul. 7 (2009), $1474-1496$.

[21] G. Plonka and D. Roşca, Easy path wavelet transform on triangulations of the sphere, Mathematical Geosciences 42(7) (2010), 839-855.

[22] G. Plonka, S. Tenorth, and D. Roşca, A hybrid method for image approximation using the easy path wavelet transform. IEEE Trans. Image Process. 20 (2011), to appear.

[23] D.D. Po and M.N. Do, Directional multiscale modeling of images using the contourlet transform, IEEE Trans. Image Process. 15 (2006), 1610-1620.

[24] R. Shukla, P.L. Dragotti, M.N. Do, and M. Vetterli, Rate-distortion optimized tree structured compression algorithms for piecewise smooth images, IEEE Trans. Image Process. 14 (2005), 343-359.

[25] M.B. Wakin, J.K. Romberg, H. Choi, and R.G. Baraniuk, Wavelet-domain approximation and compression of piecewise smooth images, IEEE Trans. Image Process. 15 (2006), 1071-108.

[26] V. Velisavljević, B. Beferull-Lozano, M. Vetterli, and P.L. Dragotti, Directionlets: anisotropic multidirectional representation with separable filtering, IEEE Trans. Image Process. 15(7) (2006), 1916-1933. 